International Journal of Pure and Applied Mathematics

Volume 114 No. 4 2017, 917-931

ISSN: 1311-8080 (printed version); ISSN: 1314-3395 (on-line version)

url: http://www.ijpam.eu

doi: 10.12732 /ijpam.v114i4.21

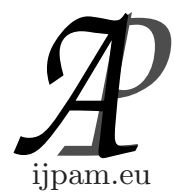

\title{
EXISTENCE, UNIQUENESS, COMPACTNESS OF THE SOLUTION SET, AND DEPENDENCE ON A PARAMETER FOR AN IMPULSIVE PERIODIC BOUNDARY VALUE PROBLEM
}

\author{
Khelifa Daoudi ${ }^{1}$, John R. Graef ${ }^{2}$, Abdelghani Ouahab ${ }^{3}$ \\ ${ }^{1,3}$ Laboratory of Mathematics \\ Sidi-Bel-Abbès University \\ P.B. 89, 22000 Sidi-Bel-Abbès, ALGERIA \\ ${ }^{2}$ Department of Mathematics \\ University of Tennessee at Chattanooga \\ Chattanooga, TN 37403-2504, USA
}

\begin{abstract}
The authors consider a second order impulsive differential equation with a parameter and periodic boundary conditions and prove results on the existence, uniqueness, compactness of the solution set, and dependence of the solutions on the parameter. Various fixed point techniques are used.
\end{abstract}

AMS Subject Classification: 34A37, 34B15, 34B37, 34K45, 39A12

Key Words: existence, uniqueness, impulsive differential equations, periodic boundary value problem, compactness of the solution set, dependence on a parameter

\section{Introduction}

The dynamics of many processes in physics, population dynamics, biology, and medicine may be subject to abrupt changes such as shocks or perturbations

Received: $\quad$ May 1, 2017

Revised: June 2, 2017

Published: June 7, 2017

${ }^{\S}$ Correspondence author
(C) 2017 Academic Publications, Ltd. url: www.acadpubl.eu 
(see for example [2] and the references therein). These perturbations may be seen as impulses. For instance, in the periodic treatment of some diseases, impulses correspond to the administration of a drug treatment. In environmental sciences, impulses correspond to seasonal changes of the water level in artificial reservoirs. Such phenomena are often modeled by impulsive differential equations and inclusions. Some good discussions and results on impulsive equations can be found in the monographs by Bainov, Lakshmikantham, and Simeonov [3], Djebali, Gorniewicz, and Ouahab [6], Graef, Henderson, and Ouahab [7], and Perestyuk, Plotnikov, Samoilenko, and Skripnik [13] and the references therein. Using fixed point arguments, existence as well as uniqueness results for some classes of impulsive boundary differential equations and dynamic equations were given by Benchohra and Eloe [4], Graef and Ouahab [9], and Nieto [12], among others.

In this paper, we consider the impulsive periodic boundary value problem with a parameter

$$
\begin{gathered}
y^{\prime \prime}-\rho^{2} y=-f(t, y, \lambda), t \in J:=[0,2 \pi], t \neq t_{k}, k=1, \ldots, m, \\
y\left(t_{k}^{+}\right)-y\left(t_{k}^{-}\right)=I_{k}\left(y\left(t_{k}^{-}\right)\right), \quad t=1, \ldots, m, \\
y^{\prime}\left(t_{k}^{+}\right)-y^{\prime}\left(t_{k}^{-}\right)=\bar{I}_{k}\left(y\left(t_{k}^{-}\right)\right), \quad t=1, \ldots, m, \\
y(0)=y(2 \pi), \quad y^{\prime}(0)=y^{\prime}(2 \pi),
\end{gathered}
$$

where $\rho \in \mathbb{R}^{+}=(0, \infty), \lambda$ is a real parameter, $f: J \times \mathbb{R} \times \mathbb{R} \rightarrow \mathbb{R}$ is a given function, $I_{k}, \bar{I}_{k} \in C(\mathbb{R}, \mathbb{R}), t_{k} \in[0,2 \pi], 0=t_{0}<t_{1}<\ldots<t_{m}<t_{m+1}=2 \pi$, and $y\left(t_{k}^{+}\right)=\lim _{h \rightarrow 0^{+}} y\left(t_{k}+h\right)$ and $y\left(t_{k}^{-}\right)=\lim _{h \rightarrow 0^{+}} y\left(t_{k}-h\right)$ represent the right and left limits of $y(t)$ at $t=t_{k}$.

One aim of this paper is to give an impulsive version of the results obtained on the problem (1), (4) considered by Graef et al. [8]. Our approach here is based on fixed point theory.

\section{Preliminaries}

In this section, we introduce notations, definitions, and preliminary facts that are used throughout the remainder of this paper. We let $C([0,2 \pi], \mathbb{R})$ be the Banach space of all continuous functions from $[0,2 \pi]$ into $\mathbb{R}$ with the norm

$$
\|x\|_{\infty}=\sup \{|x(t)|: 0 \leq t \leq 2 \pi\}
$$


and $L^{1}([0,2 \pi], \mathbb{R})$ be the Banach space of all measurable functions $x:[0,2 \pi] \rightarrow$ $\mathbb{R}$ that are Bochner integrable with the norm

$$
\|x\|_{L^{1}}=\int_{0}^{2 \pi}|x(t)| d t .
$$

Finally, $A C^{i}([0,2 \pi], \mathbb{R})$ will denote the space of $i$-times differentiable functions $y:(0,2 \pi) \rightarrow \mathbb{R}$ whose $i^{t h}$ derivative, $y^{i}$, is absolutely continuous.

We define

$$
\begin{array}{r}
P C(J, \mathbb{R})=\left\{y: J \rightarrow \mathbb{R} \mid y(t) \text { is continuous everywhere except at } t_{k}\right. \text { where } \\
\left.y\left(t_{k}^{-}\right) \text {and } y\left(t_{k}^{+}\right) \text {exist and } y\left(t_{k}^{-}\right)=y\left(t_{k}\right), k=1,2, \ldots, m\right\} .
\end{array}
$$

Clearly, $P C(J, \mathbb{R})$ is a Banach space with the norm

$$
\|y\|_{P C}=\sup \{|y(t)|: t \in J\}, y \in P C .
$$

Definition 2.1. The map $f: J \times \mathbb{R} \times \mathbb{R} \rightarrow \mathbb{R}$ is said to be $L^{1}$-Carathéodory if

(i) $t \rightarrow f(t, u, v)$ is measurable for each $u, v \in \mathbb{R}$;

(ii) $(u, v) \rightarrow f(t, u, v)$ is continuous for almost all $t \in J$;

(iii) For each $q>0$, there exists $h_{q} \in L^{1}\left(J, \mathbb{R}^{+}\right)$such that

$$
|f(t, u, \cdot)| \leq h_{q}(t) \text { for all }|u| \leq q \text { and for almost all } t \in J .
$$

\section{An Existence and Uniqueness Result}

In this section, we use Banach's fixed point theorem to prove an existence and uniqueness result for problem (1)-(4). We begin by giving the definition of a solution to this problem.

Definition 3.1. A function $y \in P C(J, \mathbb{R}) \cap A C^{1}\left(\left(t_{k}, t_{k+1}\right), \mathbb{R}\right), k=$ $0, \ldots, m$, is said to be a solution of problem (1)-(4) if $y$ satisfies equation (1) a.e. on $J$ and as well as conditions (2)-(4).

We will need the following auxiliary result. 
Lemma 3.2. The function $y \in P C(J, \mathbb{R}) \cap A C^{1}\left(\left(t_{k}, t_{k+1}\right), k=0, \ldots, m\right.$, is a solution of problem (1)-(4), if and only if $y$ is a solution of the impulsive integral equation

$$
\begin{array}{r}
y(t)=\int_{0}^{2 \pi} G(t, s) f(t, y(s), \lambda) d s-\sum_{k=1}^{m}\left[G\left(t, t_{k}\right) I_{k}\left(y\left(t_{k}\right)\right)+L\left(t, t_{k}\right) \bar{I}_{k}\left(y\left(t_{k}\right)\right)\right] \\
t \in J, \quad(6)
\end{array}
$$

where

$$
G(t, s)=\frac{1}{2 \rho\left(e^{2 \rho \pi}-1\right)} \begin{cases}e^{\rho(t-s)}+e^{\rho(2 \pi-t+s)}, & 0 \leq s \leq t \leq 2 \pi \\ e^{\rho(s-t)}+e^{\rho(2 \pi-s+t)}, & 0 \leq t \leq s \leq 2 \pi\end{cases}
$$

and

$$
L(t, s)=\frac{\partial}{\partial t} G(t, s)=\frac{1}{2\left(e^{2 \rho \pi}-1\right)} \begin{cases}e^{\rho(2 \pi-t+s)}-e^{\rho(t-s)}, & 0 \leq s<t \leq 2 \pi, \\ e^{\rho(s-t)}-e^{\rho(2 \pi-s+t)}, & 0 \leq t<s \leq 2 \pi .\end{cases}
$$

Proof. The proof is similar to one in [9] and the details will be omitted.

We now give conditions that will be needed in our main theorem in this section.

$\left(H_{1}\right)$ There exists a constant $d \geq 0$ such that

$$
|f(t, y, \lambda)-f(t, \bar{y}, \lambda)| \leq d|y-\bar{y}|
$$

for each $t \in J$ and all $y, \bar{y}, \lambda \in \mathbb{R}$.

$\left(H_{2}\right)$ There exist constants $c_{k} \geq 0$ such that

$$
\left|I_{k}(y)-I_{k}(\bar{y})\right| \leq c_{k}|y-\bar{y}|, \quad k=1, \ldots, m, \text { for all } y, \bar{y} \in \mathbb{R} .
$$

$\left(H_{3}\right)$ There exist constants $\bar{c}_{k} \geq 0$ such that

$$
\left|\bar{I}_{k}(y)-\bar{I}_{k}(\bar{y})\right| \leq \bar{c}_{k}|y-\bar{y}|, k=1, \ldots, m \text {, for all } y, \bar{y} \in \mathbb{R} .
$$

Theorem 3.3. Assume that conditions $\left(H_{1}\right)-\left(H_{3}\right)$ are satisfied and

$$
2 \pi d \sup _{(t, s) \in J \times J}|G(t, s)|+\sum_{k=1}^{m}\left(c_{k} \sup _{t \in J}\left|G\left(t, t_{k}\right)\right|+\bar{c}_{k} \sup _{t \in J}\left|L\left(t, t_{k}\right)\right|\right)<1 .
$$

Then the problem (1)-(4) has a unique solution on $J$. 
Proof. We transform the problem (1)-(4) into a fixed point problem. Consider the operator $N: P C(J, \mathbb{R}) \rightarrow P C(J, \mathbb{R})$ defined by

$$
\begin{array}{r}
N(y)(t)=\int_{0}^{2 \pi} G(t, s) f(t, y(s), \lambda) d s-\sum_{k=1}^{m}\left[G\left(t, t_{k}\right) I_{k}\left(y\left(t_{k}\right)\right)+L\left(t, t_{k}\right) \bar{I}_{k}\left(y\left(t_{k}\right)\right)\right] \\
t \in J
\end{array}
$$

As we saw in Lemma 3.2, the fixed points of $N$ are solutions to (1)-(4). We will show that $N$ is a contraction, so let $y, \bar{y} \in P C(J, \mathbb{R})$. Then, for $t \in J$ and $\lambda \in \mathbb{R}$, we have

$$
\begin{aligned}
|N(y)(t)-N(\bar{y})(t)| \leq & 2 \pi d \sup _{(t, s) \in J \times J}|G(t, s)|\|y-\bar{y}\|_{P C} \\
& +\sum_{k=1}^{m}\left[c_{k} \sup _{t \in J}\left|G\left(t, t_{k}\right)\right|+\bar{c}_{k} \sup _{t \in J}\left|L\left(t, t_{k}\right)\right|\right]\|y-\bar{y}\|_{P C} \\
\leq & {\left[2 \pi d \sup _{(t, s) \in J \times J}|G(t, s)|\right.} \\
& \left.+\sum_{k=1}^{m}\left(c_{k} \sup _{t \in J}\left|G\left(t, t_{k}\right)\right|+\bar{c}_{k} \sup _{t \in J}\left|L\left(t, t_{k}\right)\right|\right)\right]\|y-\bar{y}\|_{P C} .
\end{aligned}
$$

Thus,

$$
\|N(y)-N(\bar{y})\|_{P C} \leq \theta\|y-\bar{y}\|_{P C},
$$

where

$$
\theta=\left[2 \pi d \sup _{(t, s) \in J \times J}|G(t, s)|+\sum_{k=1}^{m}\left(c_{k} \sup _{t \in J}\left|G\left(t, t_{k}\right)\right|+\bar{c}_{k} \sup _{t \in J}\left|L\left(t, t_{k}\right)\right|\right)\right] .
$$

Since $\theta<1, N$ is a contraction. By the Banach fixed point theorem [5], $N$ has a unique fixed point in $P C(J, \mathbb{R})$, and so the problem (1)-(4) has a unique solution on $[0,2 \pi]$.

\section{Existence and Compactness of the Solution Set}

In this section, we give conditions under which the problem (1)-(4) has a solution and the set of solutions to the problem form a compact set.

Theorem 4.1. Assume that the following conditions hold: 
$\left(H_{4}\right) f: J \times \mathbb{R} \times \mathbb{R} \rightarrow \mathbb{R}$ is an Carathéodory function and $I_{k}, \bar{I}_{k} \in C(\mathbb{R}, \mathbb{R}) ;$

$\left(H_{5}\right)$ There exists $p \in L^{1}\left(J, \mathbb{R}_{+}\right)$and a constants $\alpha \in[0,1)$ such that

$$
|f(t, y, \lambda)| \leq p(t)|y|^{\alpha}, \text { for each } y, \lambda \in \mathbb{R} \text { and } t \in J
$$

$\left(H_{6}\right)$ There exist constants $d_{k}>0$ and $\alpha_{k} \in[0,1)$ such that

$$
\left|I_{k}(y)\right| \leq d_{k}|y|^{\alpha_{k}} \text { for each } y \in \mathbb{R}, k=1, \ldots, m
$$

$\left(H_{7}\right)$ There exist constants $\bar{d}_{k}>0$ and $\bar{\alpha}_{k} \in[0,1)$ such that

$$
\left|\bar{I}_{k}(y)\right| \leq \bar{d}_{k}|y|^{\bar{\alpha}_{k}} \text { for each } y \in \mathbb{R}, k=1, \ldots, m
$$

Then the problem (1)-(4) has at least one solution and the solution set

$$
S=\{y \in P C(J, \mathbb{R}): y \text { is a solution of }(1)-(4)\}
$$

is compact.

Proof. We again transform the problem (1)-(4) into a fixed point problem. Consider the operator $N$ defined in the proof of Theorem 3.3. In order to apply Schaefer's fixed point theorem [1], we first show that $N$ is completely continuous. The proof of this fact will be given in three steps.

Step 1: $N$ maps bounded sets into bounded sets in $P C(J, \mathbb{R})$.

It suffices to show that for any $q>0$ there exists a positive constant $\ell$ such that, for each $y \in B_{q}=\left\{u \in P C(J, \mathbb{R}):\|u\|_{P C} \leq q\right\}$, we have $\|N(y)\|_{P C} \leq \ell$. For each $t \in[0, b]$, we have

$$
\begin{aligned}
|N(y)(t)| \leq & \sup _{(t, s) \in J \times J}|G(t, s)| q^{\alpha} \int_{0}^{2 \pi} p(s) d s+\sum_{k=1}^{m} \sup _{t \in J}\left|G\left(t, t_{k}\right)\right| d_{k} q^{\alpha_{k}} \\
& +\sum_{k=1}^{m} \sup _{t \in J}\left|L\left(t, t_{k}\right)\right| \bar{d}_{k} q^{\bar{\alpha}_{k}} .
\end{aligned}
$$

Thus,

$$
\begin{aligned}
\|N(y)\|_{P C} \leq & \sup _{(t, s) \in J \times J}|G(t, s)| q^{\alpha}\|p\|_{L^{1}}+\sum_{k=1}^{m} \sup _{t \in J}\left|G\left(t, t_{k}\right)\right| d_{k} q^{\alpha_{k}} \\
& +\sum_{k=1}^{m} \sup _{t \in J}\left|L\left(t, t_{k}\right)\right| \bar{d}_{k} q^{\bar{\alpha}_{k}}:=\ell
\end{aligned}
$$


as desired.

Step 2: $N$ maps bounded sets into equicontinuous sets in $P C(J, \mathbb{R})$.

Let $0<\tau_{1}, \tau_{2} \in J, \tau_{1}<\tau_{2}$, and let $B_{q}$ be a bounded set in $P C(J, \mathbb{R})$ as in Step 1. If $y \in B_{q}$, then for each $t \in J$, we have

$$
\begin{aligned}
\left|N(y)\left(\tau_{2}\right)-N(y)\left(\tau_{1}\right)\right| \leq & q^{\alpha} \int_{0}^{\tau_{1}}\left[\left|G\left(\tau_{2}, s\right)-G\left(\tau_{1}, s\right)\right|\right] p(s) d s \\
& +q^{\alpha} \int_{\tau_{1}}^{\tau_{2}}\left|G\left(\tau_{2}, s\right)\right| p(s) d s \\
& +\sum_{\tau_{1}<t<\tau_{2}}\left|G\left(\tau_{2}, t_{k}\right)-G\left(\tau_{1}, t_{k}\right)\right| d_{k} q^{\alpha_{k}} \\
& +\sum_{\tau_{1}<t<\tau_{2}}\left|L\left(\tau_{2}, t_{k}\right)-L\left(\tau_{1}, t_{k}\right)\right| \bar{d}_{k} q^{\bar{\alpha}_{k}}
\end{aligned}
$$

Since the right hand side tends to zero as $\tau_{2}-\tau_{1} \rightarrow 0$, we have the equicontinuity in case $t \neq t_{i}, i=1, \ldots, m+1$. It remains to examine the equicontinuity at $t=t_{i}$.

First we prove equicontinuity at $t=t_{i}^{-}$. Fix $\delta_{1}>0$ such that $\left\{t_{k}: k \neq\right.$ i\} $\cap\left[t_{i}-\delta_{1}, t_{i}+\delta_{1}\right]=\emptyset$. For $0<h<\delta_{1}$, we have

$$
\begin{aligned}
\left|N(y)\left(t_{i}\right)-N(y)\left(t_{i}-h\right)\right| \leq & q^{\alpha} \int_{0}^{t_{i}-h}\left[\left|G\left(t_{i}, s\right)-G\left(t_{i}-h, s\right)\right|\right] p(s) d s \\
& +q^{\alpha} \int_{t_{i}-h}^{t_{i}}\left|G\left(t_{i}, s\right)\right| p(s) d s \\
& +\sum_{k=1}^{i-1}\left|G\left(t_{i}, t_{k}\right)-G\left(t_{i}-h, t_{k}\right)\right| d_{k} q^{\alpha_{k}} \\
& +\sum_{k=1}^{i-1}\left|L\left(t_{i}, t_{k}\right)-L\left(t_{i}-h, t_{k}\right)\right| \bar{d}_{k} q^{\bar{\alpha}_{k}}
\end{aligned}
$$

and we see that the right-hand side tends to zero as $h \rightarrow 0$.

To prove equicontinuity at $t=t_{i}^{+}$, fix $\delta_{2}>0$ such that $\left\{t_{k}: k \neq i\right\} \cap\left[t_{i}-\right.$ $\left.\delta_{2}, t_{i}+\delta_{2}\right]=\emptyset$. For $0<h<\delta_{2}$, we have

$$
\begin{aligned}
\left|N(y)\left(t_{i}+h\right)-N(y)\left(t_{i}\right)\right| \leq & q^{\alpha} \int_{0}^{t_{i}}\left[\left|G\left(t_{i}+h, s\right)-G\left(t_{i}, s\right)\right|\right] p(s) d s \\
& +q^{\alpha} \int_{t_{i}}^{t_{i}+h}\left|G\left(t_{i}+h, s\right)\right| p(s) d s
\end{aligned}
$$




$$
\begin{aligned}
& +\sum_{0<t_{k} \leq t_{i}}\left|G\left(t_{i}+h, t_{k}\right)-G\left(t_{i}, t_{k}\right)\right| d_{k} q^{\alpha_{k}} \\
& +\sum_{t_{i}<t \leq t_{i}+h}\left|G\left(t_{i}+h, t_{k}\right)\right| d_{k} q^{\alpha_{k}} \\
& +\sum_{0<t_{k} \leq t_{i}}\left|L\left(t_{i}+h, t_{k}\right)-L\left(t_{i}, t_{k}\right)\right| \bar{d}_{k} q^{\bar{\alpha}_{k}} \\
& +\sum_{t_{i}<t \leq t_{i}+h}\left|L\left(t_{i}+h, t_{k}\right)\right| \bar{d}_{k} q^{\bar{\alpha}_{k}},
\end{aligned}
$$

and again the right-hand side tends to zero as $h \rightarrow 0$.

Step 3: $N$ is continuous.

Let $\left\{y_{n}\right\}$ be a sequence such that $y_{n} \rightarrow y$ in $P C(J, \mathbb{R})$. Then there is an integer $q$ such that $\left|y_{n}\right| \leq q$ for all $n \in \mathbb{N}$ and $|y| \leq q$. Hence, $y_{n} \in B_{q}$ and $y \in B_{q}$. By the Lebesgue dominated convergence theorem,

$$
\begin{aligned}
\left|N\left(y_{n}\right)(t)-N(y)(t)\right| \leq & \sup _{(t, s) \in J \times J}|G(t, s)| \int_{0}^{2 \pi}\left|f\left(t, y_{n}(s), \lambda\right)-f(t, y(s), \lambda)\right| d s \\
& +\sum_{k=1}^{m} c_{k} \sup _{t \in J}\left|G\left(t, t_{k}\right)\right|\left|I_{k}\left(y_{n}\left(t_{k}\right)\right)-I_{k}\left(y\left(t_{k}\right)\right)\right| \\
& +\sum_{k=1}^{m} \bar{c}_{k} \sup _{t \in J}\left|L\left(t, t_{k}\right)\right|\left|\bar{I}_{k}\left(y_{n}\left(t_{k}\right)\right)-\bar{I}_{k}\left(y\left(t_{k}\right)\right)\right| \rightarrow 0
\end{aligned}
$$

as $n \rightarrow \infty$. Thus, $N$ is continuous.

As a consequence of Steps 1 to 3 and the Arzelà-Ascoli theorem, we conclude that $N: P C(J, \mathbb{R}) \rightarrow P C(J, \mathbb{R})$ is a completely continuous operator.

Next, we show that the set

$$
\Gamma(N)=\{y \in P C(J, \mathbb{R}): y=\bar{\lambda} N(y) \text { for some } 0<\bar{\lambda}<1\}
$$

is bounded. Let $y \in \Gamma(N)$; then $y=\bar{\lambda} N(y)$ for some $0<\bar{\lambda}<1$, Thus, for each $t \in J$,

$$
\begin{aligned}
|y(t)| \leq & \left.\int_{0}^{2 \pi} \sup _{(t, s) \in J \times J}|G(t, s)||p(s)| y(s)\right|^{\alpha} d s+\sum_{k=1}^{m} \sup _{t \in J}\left|G\left(t, t_{k}\right)\right| d_{k}\left|y\left(t_{k}\right)\right|^{\alpha_{k}} \\
& +\sum_{k=1}^{m} \sup _{t \in J}\left|L\left(t, t_{k}\right)\right| \bar{d}_{k}\left|y\left(t_{k}\right)\right|^{\bar{\alpha}_{k}},
\end{aligned}
$$


and so

$$
\begin{aligned}
\|y\|_{P C} \leq & \sup _{(t, s) \in J \times J}|G(t, s)|\|p\|_{L^{1}}\|y\|_{P C}^{\beta}+\sum_{k=1}^{m} \sup _{t \in J}\left|G\left(t, t_{k}\right)\right| d_{k}\|y\|_{P C}^{\beta} \\
& +\sum_{k=1}^{m} \sup _{t \in J}\left|L\left(t, t_{k}\right)\right| \bar{d}_{k}\|y\|_{P C}^{\beta},
\end{aligned}
$$

where $\beta=\max \left\{\alpha, \alpha_{k}, \bar{\alpha}_{k}\right\}, k=1, \ldots, m$. If $\|y\|_{P C}>1$, then we have

$$
\|y\|_{P C}^{1-\beta} \leq \sup _{(t, s) \in J \times J}|G(t, s)|\|p\|_{L^{1}}+\sum_{k=1}^{m} \sup _{t \in J}\left|G\left(t, t_{k}\right)\right| d_{k}+\sum_{k=1}^{m} \sup _{t \in J}\left|L\left(t, t_{k}\right)\right| \bar{d}_{k},
$$

and we obtain

$$
\begin{aligned}
\|y\|_{P C} \leq\left(\sup _{(t, s) \in J \times J}|G(t, s)|\|p\|_{L^{1}}\right. & +\sum_{k=1}^{m} \sup _{t \in J}\left|G\left(t, t_{k}\right)\right| d_{k} \\
& \left.+\sum_{k=1}^{m} \sup _{t \in J}\left|L\left(t, t_{k}\right)\right| \bar{d}_{k}\right)^{\frac{1}{1-\beta}}:=\psi_{*} .
\end{aligned}
$$

Therefore,

$$
\|y\|_{P C} \leq \max \left\{1, \psi_{*}\right\}:=\bar{d},
$$

so $\Gamma(N)$ is bounded. By Schaefer's fixed point theorem, $N$ has a fixed point which in turn is a solution of the problem (1)-(4).

Finally, we will show that the set

$$
S=\{y \in P C(J, \mathbb{R}): y \text { is a solution of }(1)-(4)\}
$$

is compact. Let $\left(y_{n}\right)_{n \in \mathbb{N}}$ be a sequence in $S$ and set $B=\left\{y_{n}: n \in \mathbb{N}\right\} \subseteq$ $P C(J, \mathbb{R})$. From earlier parts of the proof of this theorem, we see that $B$ is bounded and equicontinuous. Then, from the Ascoli-Arzelà theorem, we can conclude that $B$ is compact. Hence, $\left(y_{n}\right)_{n \in \mathbb{N}}$ has a subsequence $\left(y_{n_{m}}\right)_{n_{m} \in \mathbb{N}} \subseteq S$ that converges to some $y$. Let

$$
z_{0}(t)=\int_{0}^{2 \pi} G(t, s) f(t, y(s), \lambda) d s+\sum_{k=1}^{m} G\left(t, t_{k}\right) I_{k}\left(y\left(t_{k}\right)\right)+\sum_{k=1}^{m} L\left(t, t_{k}\right) \bar{I}_{k}\left(y\left(t_{k}\right)\right) .
$$

Then,

$$
\left|y_{n_{m}}-z_{0}(t)\right| \leq \int_{0}^{2 \pi}|G(t, s)|\left|f\left(t, y_{n_{m}}(s), \lambda\right)-f(t, y(s), \lambda)\right| d s
$$




$$
\begin{aligned}
& +\sum_{k=1}^{m}\left|G\left(t, t_{k}\right)\right|\left|I_{k}\left(y_{n_{m}}\left(t_{k}\right)\right)-I_{k}\left(y\left(t_{k}\right)\right)\right| \\
& +\sum_{k=1}^{m}\left|L\left(t, t_{k}\right)\right|\left|\bar{I}_{k}\left(y_{n_{m}}\left(t_{k}\right)\right)-\bar{I}_{k}\left(y\left(t_{k}\right)\right)\right| .
\end{aligned}
$$

As $n_{m} \rightarrow \infty, y_{n_{m}} \rightarrow z_{0}(t)$, and so

$$
y(t)=\int_{0}^{2 \pi} G(t, s) f(t, y(s), \lambda) d s+\sum_{k=1}^{m} G\left(t, t_{k}\right) I_{k}\left(y\left(t_{k}\right)\right)+\sum_{k=1}^{m} L\left(t, t_{k}\right) \bar{I}_{k}\left(y\left(t_{k}\right)\right) .
$$

Hence, $y \in S$ and so $S$ is compact.

\section{Dependence on the Parameter}

In addition to determining additional conditions for the existence of positive solutions, in this section we also wish to examine their dependence on the parameter $\lambda$ (see Theorem 5.5 below). Difficulties arise in this analysis due to the fact that, although the Green's function $G(t, s)$ is positive, $L(t, s)=$ $\frac{\partial}{\partial t} G(t, s)$ does not have fixed sign and is not continuous at $t=s$. Hence, in this section we consider a special case of problem (1)-(4), namely,

$$
\begin{gathered}
y^{\prime \prime}-\rho^{2} y=-\lambda g(t) h(y), t \in J:=[0,2 \pi], t \neq t_{k}, k=1, \ldots, m, \\
y\left(t_{k}^{+}\right)-y\left(t_{k}^{-}\right)=I_{k}\left(y\left(t_{k}^{-}\right)\right), \quad t=1, \ldots, m, \\
y(0)=y(2 \pi), \quad y^{\prime}(0)=y^{\prime}(2 \pi),
\end{gathered}
$$

where $g: J \rightarrow \mathbb{R}$ and $h: \mathbb{R} \rightarrow \mathbb{R}$ are continuous functions.

We begin with a lemma that ensures the existence of a unique positive solution to our problem.

Lemma 5.1. In addition to conditions $\left(H_{1}\right)-\left(H_{3}\right)$ and (9) assume that:

$\left(H_{8}\right) g(t) \geq 0$ for $t \in J$ and $h(y) \geq 0$ for $y \in \mathbb{R}$.

$\left(H_{9}\right) I_{k}(y) \leq 0$, for each $y \in \mathbb{R}$ and $k=1, \ldots, m$.

Then the problem (11)-(13) has a unique positive solution on $J$. 
Proof. We transform problem (11)-(13) into a fixed point problem by considering the operator $N: P C(J, \mathbb{R}) \rightarrow P C(J, \mathbb{R})$ defined by

$$
N(y)(t)=\lambda \int_{0}^{2 \pi} G(t, s) g(s) h(y(s)) d s-\sum_{k=1}^{m}\left[G\left(t, t_{k}\right) I_{k}\left(y\left(t_{k}\right)\right)\right], \quad t \in J .
$$

To show that the fixed points of $N$ are positive solutions to (11)-(13), let $y \in P C(J, \mathbb{R})$ be a fixed point of $N$. It is clear that

$$
y(t)=\lambda \int_{0}^{2 \pi} G(t, s) g(s) h(y(s)) d s-\sum_{k=1}^{m}\left[G\left(t, t_{k}\right) I_{k}\left(y\left(t_{k}\right)\right)\right], \quad t \in J,
$$

(see (6)) which implies that $y$ is a solution of $(1)-(4)$.

If $y$ is a fixed point of $N$, then $\left(H_{8}\right)-\left(H_{9}\right)$ imply that $y(t) \geq 0$ for each $t \in J$.

As in the proof of Theorem 3.3, we can show that $N$ is contraction. So by Banach's fixed point theorem [5], $N$ has a unique fixed point $y$ that is a positive solution of (11)-(13).

Definition 5.2. Let $(E,\|\|$.$) be a real Banach space. A nonempty, closed,$ convex set $C \subset E$ is said to be a cone provided the following conditions are satisfied:

(a) if $y \in C$ and $\lambda \geq 0$, then $\lambda y \in C$;

(b) if $y \in C$ and $-y \in C$, then $y=0$.

We say that $C$ is a solid cone if $C^{\circ}$ is not empty, where $C^{\circ}$ is the interior of $C$.

Every cone $C \subset E$ induces a partial ordering, " $\leq$ " on $E$ defined by

$$
x \leq y \quad \text { if and only if } y-x \in C \text {. }
$$

Definition 5.3. Let $C$ be a solid cone in a real Banach space $E$ and let $N: C^{\circ} \rightarrow C^{\circ}$ be an operator. We say that $N$ is an $\alpha$-concave operator ( $\alpha$-convex operator) if there exists $0 \leq \alpha<1$ such that

$$
N(t x) \geq t^{\alpha} N x \quad\left(N(t x) \geq t^{-\alpha} N x\right) \quad \text { for any } \quad x \in C^{\circ} \text { and } 0<t<1 .
$$

Lemma 5.4. ([10]) Let $N$ be a normal solid cone in a real Banach space, $N: C^{\circ} \rightarrow C^{\circ}$ be an $\alpha$-concave increasing (or $\alpha$-convex decreasing) operator. Then $N$ has only one fixed point in $C^{\circ}$.

Theorem 5.5. In addition to conditions $\left(H_{8}\right)-\left(H_{9}\right)$, assume that the following conditions are satisfied: 
(i) $h$ is nondecreasing;

(ii) $I_{k}, k=1, \ldots, m$, are decreasing functions;

(iii) $h(\eta u) \geq \eta^{\alpha} h(u)$ and $I_{k}(\eta u) \leq \eta^{\alpha} I_{k}(u)$ for any $0<\eta<1$, where $0 \leq \alpha<$ 1.

Then the problem (11)-(13) has a unique positive solution $y_{\lambda}(t)$. Furthermore, such a solution $y_{\lambda}(t)$ satisfies the following properties:

(j) $y_{\lambda}(t)$ is increasing in $\lambda$, i.e., $\lambda_{1}>\lambda_{2}>0$ implies $y_{\lambda_{1}}(t)>y_{\lambda_{2}}(t)$ for $t \in J$;

(jj) $\lim _{\lambda \rightarrow 0^{+}}\left\|y_{\lambda}(t)\right\|=0$ and $\lim _{\lambda \rightarrow+\infty}\left\|y_{\lambda}(t)\right\|=+\infty$ for any $t \in J$;

(jjj) $y_{\lambda}(t)$ is continuous with respect to $\lambda$, i.e., if $\lambda \rightarrow \lambda_{0}>0$, then $\| y_{\lambda}(t)-$ $y_{\lambda_{0}}(t) \| \rightarrow 0$ for any $t \in J$.

Proof. Let

$$
C=\{y \in P C(J, \mathbb{R}): y(t) \geq 0 \text { for } t \in J\}
$$

be our cone in $P C(J, \mathbb{R})$. Then $\left(H_{8}\right)$ and $\left(H_{9}\right)$ imply that $N(C) \subset C$, and it is easy to see that $N: C^{\circ} \rightarrow C^{\circ}$. We assert that $N: C^{\circ} \rightarrow C^{\circ}$ is an $\alpha$-concave increasing operator. Indeed

$$
\begin{aligned}
N(\eta y) & =\lambda \int_{0}^{2 \pi} G(t, s) g(s) h(\eta y(s)) d s-\sum_{k=1}^{m}\left[G\left(t, t_{k}\right) I_{k}\left(\eta y\left(t_{k}\right)\right)\right] \\
& \geq \eta^{\alpha}\left(\lambda \int_{0}^{2 \pi} G(t, s) g(s) h(y(s)) d s-\sum_{k=1}^{m}\left[G\left(t, t_{k}\right) I_{k}\left(y\left(t_{k}\right)\right)\right]\right) \\
& \geq \eta^{\alpha} N(y) \quad \text { for any } 0<\eta<1,
\end{aligned}
$$

where $0 \leq \alpha<1$. By (i) and (ii),

$$
\begin{aligned}
N\left(y_{*}\right)(t) & =\lambda \int_{0}^{2 \pi} G(t, s) g(s) h\left(y_{*}(s)\right) d s-\sum_{k=1}^{m}\left[G\left(t, t_{k}\right) I_{k}\left(y_{*}\left(t_{k}\right)\right)\right] \\
& \leq \lambda \int_{0}^{2 \pi} G(t, s) g(s) h\left(y_{* *}(s)\right) d s-\sum_{k=1}^{m}\left[G\left(t, t_{k}\right) I_{k}\left(y_{* *}\left(t_{k}\right)\right)\right] \\
& =N\left(y_{* *}\right)(t) \text { for } y_{*}, y_{* *} \in C \text { with } y_{*} \leq y_{* *} .
\end{aligned}
$$

In view of Lemma $5.4, N$ has a unique fixed point $y_{\lambda} \in C^{\circ}$. Using exactly the same argument as in the second part of the proof of [10, Theorem 6], we can show that $(j),(j j)$, and $(j j j)$ hold, and we omit the details. This completes the proof of the theorem. 
Theorem 5.6. Assume that conditions $\left(H_{4}\right)-\left(H_{6}\right)$ and $\left(H_{8}\right)-\left(H_{9}\right)$ hold. Then the problem (11)-(13) has at least one positive solution on $J$.

Proof. First we transform problem (11)-(13) into a fixed point problem using the operator $N$ defined in (14). As in the proof of Lemma 5.1, we can show that the fixed points of $N$ are positive solutions of (1)-(4). As in the proof of Theorem 4.1, we can use Schaefer's fixed point theorem [1] to show that $N$ does in fact have a fixed point. This proves the theorem.

Next, we obtain two solutions of problem (1)-(4) using Krasnosel'skii's twin fixed point theorem [11]. This will employ the use of an appropriate cone.

Theorem 5.7. In addition to $\left(H_{4}\right)-\left(H_{6}\right)$ and $\left(H_{8}\right)-\left(H_{9}\right)$, assume that:

$\left(H_{10}\right)$ There exist $R>0$ and $r>0$, with $r<R$, such that

$$
\lambda \sup _{(t, s) \in J \times J}|G(t, s)|\|g\|_{\infty} h^{*}(r)+\sum_{k=1}^{m} \sup _{t \in J}\left|G\left(t, t_{k}\right)\right| d_{k} r^{\alpha_{k}}<r,
$$

where $h^{*}(r)=\sup _{u \in(0, r]}|h(u)|$, and

$$
\min _{t \in[0,2 \pi]}\left(\lambda \int_{0}^{2 \pi} G(t, s) g(s) h(w(s)) d s-\sum_{k=1}^{m} G\left(t, t_{k}\right) I_{k}\left(w\left(t_{k}\right)\right)\right)>R \text { if } w>r .
$$

Then the problem (11)-(13) has at least has at least two positive solutions $y_{1}$ and $y_{2}$ such that $\left\|y_{1}\right\|<r$ and $r<\left\|y_{2}\right\| \leq R$.

Proof. Let $C$ be the cone defined in (16). Then $\left(H_{8}\right)-\left(H_{9}\right)$ imply that $N(C) \subset C$. For any $R>0$, let

$$
C_{R}=\{y \in C:\|y\|<R\} .
$$

Using $\left(H_{4}\right)-\left(H_{6}\right)$, we can show that $N: C_{R} \rightarrow C$ is a completely continuous operator.

Now it remains to show that the hypotheses of Krasnosel'skii's twin fixed point theorem [11] are satisfied.

Claim 1: $\|N(y)\|_{P C}<\|y\|_{P C}$ for all $y \in \partial C_{r}$, where $C_{r}=\{y \in C:\|y\|<r\}$.

Now for $y \in \partial C_{r}$, we have $\|y\|_{P C}=r$, and from $\left(H_{6}\right)$ and $\left(H_{10}\right)$,

$$
|N(y)(t)| \leq \lambda \int_{0}^{2 \pi}|G(t, s)||g(s) h(u(s))| d s+\sum_{k=1}^{m}\left|G\left(t, t_{k}\right)\right|\left|I_{k}\left(y\left(t_{k}\right)\right)\right|
$$




$$
\begin{aligned}
& \leq \lambda \sup _{(t, s) \in J \times J}|G(t, s)|\|g\|_{\infty} h^{*}(r)+\sum_{k=1}^{m} \sup _{t \in J}\left|G\left(t, t_{k}\right)\right| d_{k} r^{\alpha_{k}} \\
& <r=\|y\|_{P C} .
\end{aligned}
$$

Hence,

$$
\|N(y)\|_{P C}<\|y\|_{P C} \text { for each } y \in \partial C_{r} .
$$

Claim 2: $\|N(y)\|_{P C}>\|y\|_{P C}$ for all $y \in \partial C_{R}$.

For $y \in \partial C_{R}$, we have $\|y\|_{P C}=R$, so $r<\|y\|_{P C}=R$, and for $w>r$, from $\left(H_{10}\right)$ we have

$$
\begin{aligned}
N(y)(t) & \geq \min _{t \in[0,2 \pi]}\left(\lambda \int_{0}^{2 \pi} G(t, s) g(s) h(w(s)) d s-\sum_{k=1}^{m} G\left(t, t_{k}\right) I_{k}\left(w\left(t_{k}\right)\right)\right) \\
& >R=\|y\|_{P C} .
\end{aligned}
$$

Therefore,

$$
\|N(y)\|_{P C}>\|y\|_{P C} \text { for each } y \in \partial C_{R} .
$$

Thus, the problem (11)-(13) has at least has at least two positive solutions $y_{1}$, $y_{2}$ such that $\left\|y_{1}\right\|<r$ and $r<\left\|y_{2}\right\| \leq R$.

\section{References}

[1] R. P. Agarwal, M. Meehan, and D. O'Regan, Fixed Point Theory and Applications, Cambridge Tracts in Mathematics, 141. Cambridge University Press, Cambridge, 2001.

[2] Z. Agur, L. Cojocaru, G. Mazaur, R. M. Anderson, and Y. L. Danon, Pulse mass measles vaccination across age cohorts, Proc. Nat. Acad. Sci. USA 90 (1993), 11698-11702.

[3] D. D. Bainov, V. Lakshmikantham, and P. S. Simeonov, Theory of Impulsive Differential Equations, World Scientific, Singapore, 1989.

[4] M. Benchohra and P. Eloe, On nonresonance impulsive functional differential equations with periodic boundary conditions, Appl. Math. E-Notes 1 (2001), 65-72.

[5] K. Deimling, Nonlinear Functional Analysis, Springer-Verlag, Berlin, 1985.

[6] S. Djebali, L. Gorniewicz, and A. Ouahab, Solutions Sets for Differential Equations and Inclusions, De Gruyter Series in Nonlinear Analysis and Applications Vol. 18, De Gruyter, Berlin, 2013.

[7] J. R. Graef, J. Henderson and A. Ouahab, Impulsive Differential Inclusions, A Fixed Point Approach, De Gruyter Series in Nonlinear Analysis and Applications Vol. 20, De Gruyter, Berlin, 2013.

[8] J. R. Graef, L. Kong, and H. Wang, Existence, multiplicity, and dependence on a parameter for a periodic boundary value problem, J. Differential Equations 245 (2008), 1185-1197. 
[9] J. R. Graef and A. Ouahab, Nonresonance impulsive functional dynamic equations on times scales, Int. J. Appl. Math. Sci. 2 (2005), 65-80.

[10] D. J. Guo, The fixed point and eigenvalue of a class of concave and convex operator, Chinese Sci. Bull. 15 (1985), 1132-1135 (in Chinese).

[11] S. Heikkilä and V. Lakshmikantham, Monotone Iterative Techniques for Discontinuous Nonlinear Differential Equations, Monographs and Textbooks in Pure and Applied Mathematics, Vol. 181, Dekker, New York, 1994.

[12] J. J. Nieto, Basic theory for nonresonance impulsive periodic problems of first order, $J$. Math. Anal. Appl. 205 (1997), 423-433.

[13] N. Perestyuk, V. Plotnikov, M. Samoilenko, and V. Skripnik, Differential Equations with Impulse Effects: Multivalued Right-hand Sides with Discontinuities, De Gruyter GmbH and Co. KG, Berlin/Boston, 2011. 
